\title{
Evolution of Clouds of Migrating Micro-particles with Hydrodynamic and Electrostatic Interactions
}

\author{
Sheng Chen ${ }^{1}$ and Shuiqing $\mathrm{Li}^{1, \star}$ \\ ${ }^{1}$ Key Laboratory for Thermal Science and Power Engineering of Ministry of Education, Department of Thermal Engineering, \\ Tsinghua University, Beijing 100084,China
}

\begin{abstract}
The evolution of a migrating cloud of particles with electrohydrodynamic interactions is numerically investigated. The hydrodynamic interaction is modelled by Oseen dynamics in the limit of small-but-finite particle Reynolds number. The effects of external field and the particle-particle Coulomb repulsion, calculated through pairwise summation, are quantified by a charge parameter, $\kappa_{q}$. With a small or zero $\kappa_{q}$, the cloud is seen to flatten into a toroidal configuration and eventually breaks up into two small clouds, indicating that the external electrostatic field has a similar effect with gravity. Increasing the long-range Coulomb repulsion can delay or even totally prevent the breakup. With sufficiently strong repulsion, the cloud undergoes a self-similar expansion. Finally, we show that the breakup of the cloud is strongly related to the initial inhomogeneity of the particle concentration. This chaotic characteristic, however, is dramatically depressed by the long-range Coulomb repulsion.
\end{abstract}

\section{Introduction}

The migration of charged particles in an electrostatic field is ubiquitous in engineering processes, including dust removal in an electrostatic precipitator [1,2], films fabrication using electrophoretic deposition [3] and self-assembly of colloidal particles [4]. During these process, both hydrodynamic and electrostatic interactions between multiple particles result in a wealth of complex collective behaviours of the particle clouds.

For flows with a sufficiently low Reynolds number, the hydrodynamic interaction among particles is usually simulated by solving the Stokes/Oseen equations. The disturbance of every individual particle on the flow field is linearly summed to obtain the flow field at the position of other particles [5, 6]. Stokes dynamics has been used to investigate the evolution of the settling velocity and the shape of a particle cloud during gravity settling in conditions where inertia is negligible [7]. Cloud settling in the case of small-but-finite Reynolds number was investigated by Pignatel et al. It is proposed that the evolution of the cloud deformation is accelerated as the inertia is increased [8]. Yang et al. proposed analytical expressions of settling velocities for both spherical and columnar particle clouds, and compared them with the result of Stokes clouds to reflect the effect of the fluid inertia [9].

Most studies are focused on cloud settling under gravity, while the migrating of charged particles in an electrostatic field is rarely involved. The biggest difference between the electrostatic migration process and the gravitational settling process is the need to account for the

\footnotetext{
^e-mail: lishuiqing@tsinghua.edu.cn
}

inter-particle Coulomb repulsion. This long-range repulsion has been found to have significant effects on particle packing structure [10]. However, the migration behavior of particle clouds with a coupling effect of hydrodynamic/electrostatic interactions still remains unclear. In this paper, we try to understand this process in the limit of small-but-finite particle Reynolds number through Oseen simulations with special attention paid to the effect of long-range Coulomb repulsion.

\section{Models and Methods}

\subsection{Simulation conditions}

A sketch of the simulated system is shown in Fig. 1. A spherical cloud with radius $R_{0}$ containing $N=1000$ charged particles is immersed in an unbounded fluid of viscosity $\mu_{f}$. The fluid is at rest at infinity and the cloud is migrating under an action of a uniform electric field. The radius $r_{p}$, density $\rho_{p}$, charge $q_{0}$ of particles and the strength of the electric field intensity $E_{0}$ are also listed in Fig. 1. Besides the hydrodynamic interactions (modelled by Oseen dynamics), we also consider the long-rang electrostatic forces: a uniform external electrostatic force in $x$ direction $\left(E_{0} q_{0}\right)$ and a pairwise Coulomb repulsion $\left(q_{0}^{2} / 4 \pi \varepsilon r_{i j}^{2}\right)$. It should be mentioned that, higher-order multipoles, e.g., dipoles or quadrupoles, decay sufficiently fast with the distance and are ignored in this work due to the large separation between particles. Such interactions, which may have effects on particles dynamic when the concentration of particles in the cloud is high, will be left to future work. During our simulation, the information of 


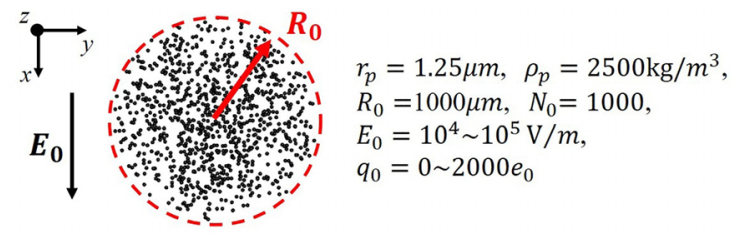

Figure 1. Sketch of the simulated system and main parameters.

all particles (position, velocity and forces) and flow field (velocity and pressure) are simultaneously recorded. Thus the temporal evolution of migrating cloud can be easily captured.

\subsection{Oseen Dynamics}

For a particle with radius $r_{p}$ translating with a velocity $U_{S}$ relative to the surrounding fluid, the generated flow field can be written as:

$$
\begin{aligned}
u_{r}= & \frac{U_{S} r_{p}^{2}}{r^{2}}\left\{-\frac{r_{p}}{2 r} \cos \theta-\frac{3(1-\cos \theta) r}{4 r_{p}} \exp \left(-\frac{\operatorname{Re} e_{S} r(1+\cos \theta)}{2 r_{p}}\right)\right. \\
& \left.+\frac{3}{2 R e_{S}}\left[1-\exp \left(-\frac{R e_{S} r(1+\cos \theta)}{2 r_{p}}\right)\right]\right\} \\
u_{\theta}= & -\frac{U_{S} r_{p}}{r} \sin \theta\left[\frac{r_{p}^{2}}{4 r^{2}}+\frac{3}{4} \exp \left(-\frac{R e_{S} r(1+\cos \theta)}{2 r_{p}}\right)\right] .
\end{aligned}
$$

With the polar axis $(\theta=0)$ coincident with the direction of particle motion. $R e_{S}=2 r_{p} U_{S} \rho_{f} / \mu_{f}$ is the instantaneous particle's Reynolds number based on particle slip velocity $U_{S}$. Since the particle Stokes number is very small $\left(\sim 10^{-2}\right)$ in the current simulations, it is reasonable to assume that the particle inertia is negligible. Thus $\mathbf{U}_{S}$ can be directly calculated from the external forces acting on the particle through $\mathbf{U}_{S}=\mathbf{F}_{e x t} / 6 \pi \mu_{f} r_{p}$. Then the velocity for a particle $i$ inside the cloud can be obtained as a summation of its slip velocity $\mathbf{U}_{S, i}$ and the fluid velocity $\mathbf{u}_{i}$ at its centre, which is solved by a matrix equation $\mathbf{u}_{i}=\sum_{j \neq i} \mathbf{W}_{i j} \mathbf{U}_{S, j}$ once the slip velocity of each particle is known.

In this work, the electrostatic forces are the only external forces and the equation of motion for $i^{\text {th }}$ particle can be expanded as

$$
\begin{aligned}
\mathbf{v}_{i} & =\mathbf{u}_{i}+\mathbf{U}_{S, i} \\
& =\mathbf{u}_{i}+\frac{1}{6 \pi \mu_{f} r_{p}}\left(\mathbf{E}_{0} q_{i}+q_{i} \sum_{j \neq i} \frac{q_{j} \mathbf{r}_{i j}}{4 \pi \varepsilon_{0} r_{i j}^{3}}\right) .
\end{aligned}
$$

Here, $\mathbf{E}_{0}$ is the external field in $x$ direction and $q_{i}$ is the charge on particles which is identical to $q_{0}$ in this work. Normalizing the velocity by the migrating velocity of an isolated particle $U_{0}=E_{0} q_{0} / 6 \pi \mu_{f} r_{p}$, the length by cloud radius $R_{0}$, and the time by $R_{0} / U_{0}$, Eq. 2 becomes

$$
\hat{\mathbf{v}}_{i}=\hat{\mathbf{u}}_{i}+\left(1 \mathbf{e}_{x}+\kappa_{q} \frac{1}{N} \sum_{j \neq i} \frac{\hat{\mathbf{r}}_{i j}}{\hat{r}_{i j}^{3}}\right) .
$$

Several dimensionless parameters influence migrating process. The most important one is particle Reynolds number $R e_{p}=E_{0} q_{0} \rho_{f} / 6 \pi \mu_{f}^{2}$, which has been related to the electrostatic properties. Another dimensionless parameter indicated by Eq. 3 is the charge parameter, $k_{q}$, which is defined as

$$
\kappa_{q}=\frac{q_{0} N}{4 \pi \varepsilon_{0} E_{0} R_{0}^{2}} .
$$

$\kappa_{q}$ is interpreted as the ratio of the order of magnitude of particle-particle Coulomb repulsion to that of uniform external force.

\section{Results and discussions}

\subsection{Clouds evolutions}

The typical evolution of a cloud with different strength of Coulomb repulsion $\left(\kappa_{q}\right)$ is shown in Fig. 2. The cloud with $\kappa_{q}=0$ (Fig. 2(A)), is seen to flatten (at dimensionless time $\hat{t}=15)$ and to transit into a toroidal shape $(\hat{t}=30)$. Then the toroidal cloud expands and finally breaks up into two small clouds $(\hat{t}=60)$ which may undergo repeating beakup. This is qualitatively in accord with the simulation and the experiments of settling under gravity [7,8]. The result indicates that the external electrostatic field has a similar effect with gravity.

In the presence of long-range Coulomb repulsion (Fig. 2(B)), the vertical collapse of the cloud still remains. However, instead of an immediate breakup at $\hat{t}=30$, the cloud remains in an oblate shape. Breakup is delayed to $\hat{t}=60$. When $\kappa_{q}$ further increases, the cloud undergoes a self-similar expansion (Fig. 2(C). No breakup happens during a long-term observation $(\hat{t} \sim 100)$. These results suggest that the long-range repulsion makes the cloud more stable during its migration.

\subsection{Migrating velocity and aspect ratio}

The effect of the long-range repulsion can be further quantified by the temporal evolution of the migrating velocity and the aspect ratio of the clouds (Fig. 3). The migrating velocity has been normalized by the migrating velocity of an isolated particle. The initial migrating velocity at $\hat{t}=0$ is $U_{x} / U_{0}=2.13$, which accords well with the analytical prediction $U_{x} / U_{0}=1.2\left(r_{p} / R_{0}\right) N f\left(l^{*}\right)+1=2.12$ of cloud settling under gravity [9]. This result indicates that the uniform electrostatic force plays a similar role as gravity. And the prediction of Yang et al.[9] still holds after substituting the migrating velocity of a charged particle $\left(E_{0} q_{0} / 6 \pi \mu_{f} r_{p}\right)$ for the terminal settling velocity of a particle under gravity $\left(m_{p} g / 6 \pi \mu_{f} r_{p}\right)$.

The decreasing trends of $U_{x} / U_{0}$ for the three cases are quite similar, however the underlying physical mechanism is different. For the cloud without long-range repulsion, the drop of the $U_{x} / U_{0}$ is mainly due to the evolution towards a torus and the following breakup, which 


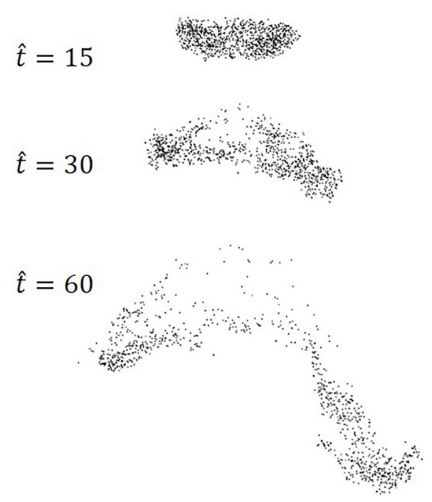

(A) $\kappa_{q}=0$
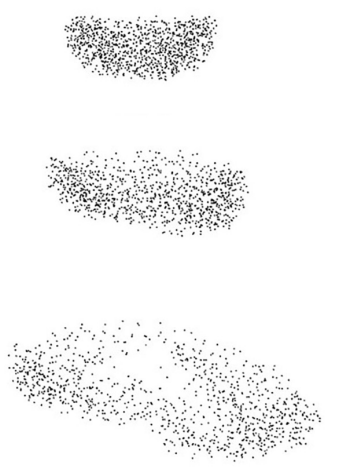

(B) $\kappa_{q}=0.023$
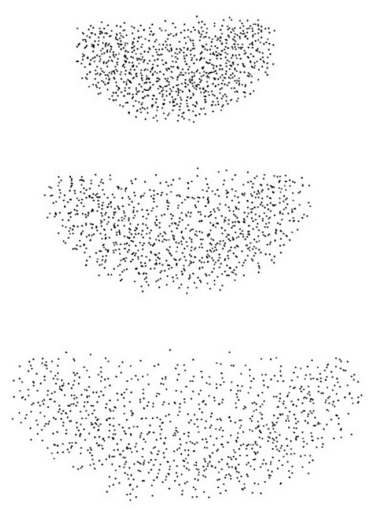

(C) $\kappa_{q}=0.14$

Figure 2. Typical evolution of clouds with $R e_{p}=1.27 \times 10^{-3}$ and (A) $\kappa_{q}=0,(\mathrm{~B}) \kappa_{q}=0.023$ and $(\mathrm{C}) \kappa_{q}=0.14$.

splits the big cloud into small clouds and weakens the particle-particle hydrodynamic interaction (Fig. 2(A)). As for clouds with long-range repulsion, despite the absence of any breakups, a radial expansion also leads to a decrease of hydrodynamic interaction resulting a smaller migrating velocity. And the decrease rate of $U_{x} / U_{0}$ is larger for cloud with a higher $\kappa_{q}$.

The temporal evolution of the aspect ratio is a more intuitive way to reflect the difference between migrating clouds with and without long-range repulsion. The aspect ratio is defined as $\lambda=R_{h} / R_{v}$, where the horizontal radius $R_{h}$ is defined as the average of the maximum distance from the centre of mass over four quadrants in the horizontal plane and the vertical radius $R_{v}$ is the distance form the leading particle to the center of mass of the cloud. As shown in Fig. 3(B), the initial increase of $\lambda$ is caused by the vertical collapse and then remarkably difference can be observed. For cloud without long-range repulsion $\left(\kappa_{q}=0\right), \lambda$ rapidly decreases from the peak (a) at $\hat{t}=17$ to the point (b) at $\hat{t}=33$ (the corresponding snapshots are shown below the figure). It is caused by the breakup of the cloud. Once two small clouds are formed, the inhomogeneity of the distribution of particles inside the two clouds causes a obvious difference between their migrating velocities. Consequently, they separate in the vertical direction, which leads to a increase of $R_{v}$ i.e. a smaller $\lambda$. To some extent, the increasing-decreasing trend of $\lambda \sim \hat{t}$ can be regarded as a sign of cloud's destabilization.

In the presence of long-range repulsion, the decrease of $\lambda$ is delayed $\left(\kappa_{q}=0.023\right)$ or even completely disappeared $\left(\kappa_{q}=0.14\right)$. This result suggests that the longrange Coulomb repulsion can help the cloud maintain a stable configuration.

\subsection{Stability of migrating clouds}

In this section, we briefly discuss the stability of migrating clouds. It has been reported that long-range hydro-
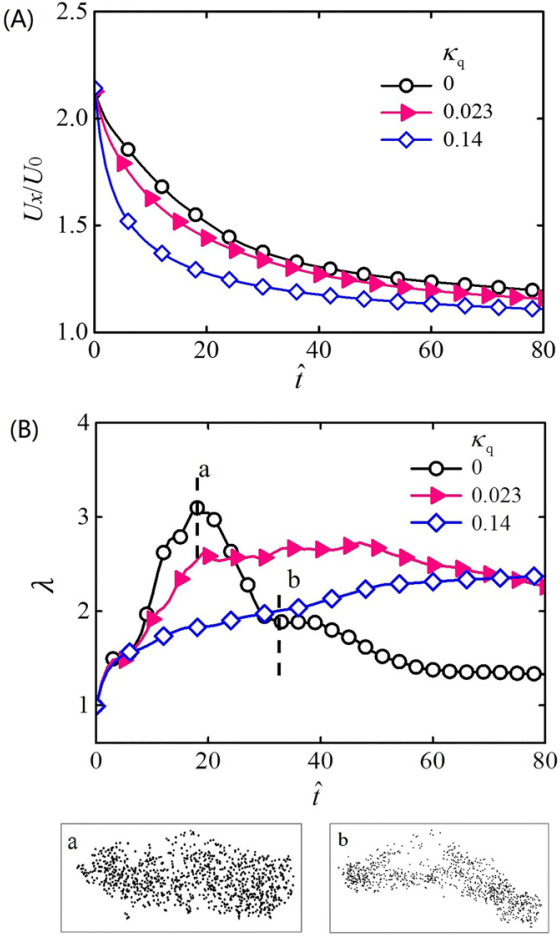

Figure 3. (A) Normalized cloud migrating velocity $U_{x} / U_{0}$ and (B) aspect ratio $\lambda$ versus time $\hat{t}$. The snapshots below are taken at (a) $\hat{t}=17$ and (b) $\hat{t}=33$ in the case of $\kappa_{q}=0$.

dynamic interactions lead to a complex chaotic dynamics and the evolution of a migrating cloud is extreme sensitive to initial configurations[7, 11]. Therefore, we plot the angular probability distribution of particles in the cloud at three typical moment $\hat{t}=0,17$ and 33 to show the relationship between initial configurations and clouds' destabilization. As shown in Fig. 4(A), for the case without Coulomb repulsion, the non-uniformity in the angular dis- 

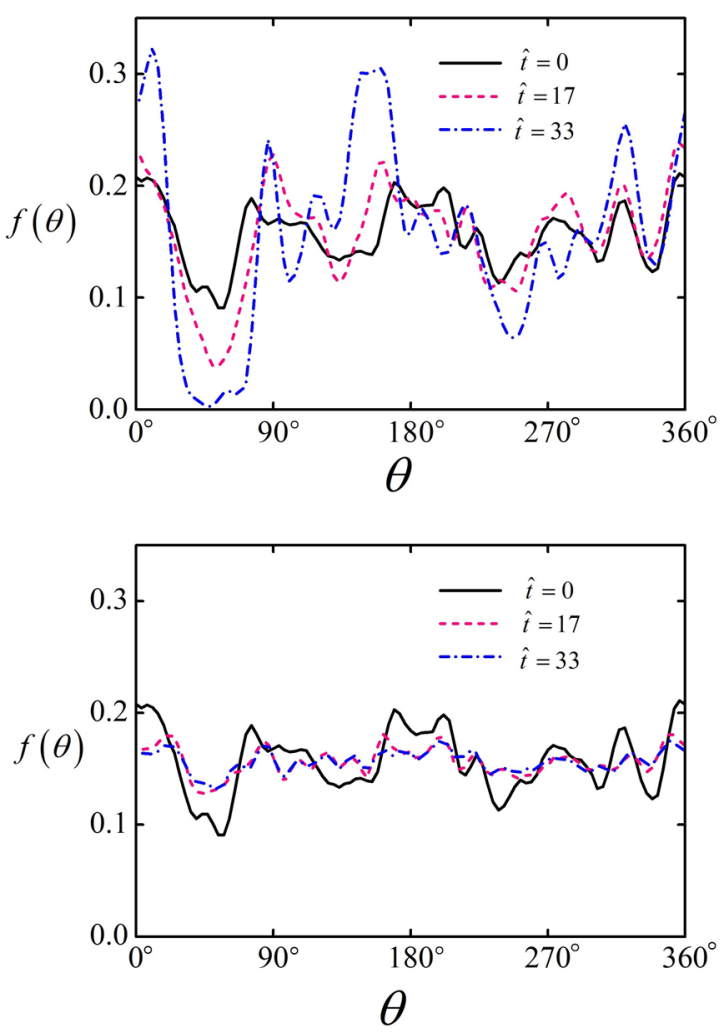

Figure 4. Angular probability distribution of particles in a cloud with (A) $\kappa_{q}=0$ and (B) $\kappa_{q}=0.14$ at $\hat{t}=0,17$ and 33 .

tribution is enhanced with time. At $\hat{t}=33$, the cloud is drastically stretched in the directions with higher particle concentrations (around $0^{\circ}$ and $150^{\circ}$ ). In these particle-rich regions, a higher local concentration leads to a stronger hydrodynamic interaction and a larger local migrating velocity, which eventually induce the breakup. In this regard, we infer that for the migrating cloud, which has a strong chaotic characteristic, the breakup phenomenon is strongly related to the inhomogeneity of initial particle distribution.

On the other hand, for the cloud with a sufficiently strong long-range repulsion $\left(\kappa_{q}=0.14\right)$, the initial inhomogeneity is obviously reduced during the migration. The angular probability distributions at $\hat{t}=17$ and $\hat{t}=33$ are essentially identical and are close to the average value $(1 / 2 \pi \approx 0.159)$. These results suggest that, an alteration of micro-scale interaction can cause a significant change in the macro behaviour of a particle system. To be specific here, the long-range Coulomb repulsion increases the stability of migrating clouds.

\section{Conclusions}

In summary, we use Oseen dynamic simulation with electrostatic interactions taken into account to investigate the electrohydrodynamic phenomenon of a migrating particle cloud. It is observed that, the long-range Coulomb repulsion can delay or even totally prevent the breakup of the cloud. Entirely different evolutions of the aspect ratio $\lambda$ are demonstrated: an increasing-decreasing trend of $\lambda$, which can be regarded as a sign of destabilization, is observed for cloud without long-range repulsion, while a cloud with strong Coulomb repulsion undergoes a self-similar expansion with a slowly changing $\lambda$. Finally, we show that the breakup of the cloud is strongly related to the initial inhomogeneity of the particle concentration. This chaotic characteristic of a migrating cloud is dramatically depressed by the long-range Coulomb repulsion.

\section{Acknowledgements}

This work has been funded by the National Key Research and Development Program (Grant No. 2016YFB0600602) and the National Natural Science Funds of China (No. 51390491)

\section{References}

[1] J.S. Marshall, S. Li, Adhesive Particle Flows (Cambridge University Press, 2014)

[2] M. Yang, S. Li, G. Liu, Q. Yao, Electricallyenhanced deposition of fine particles on a fiber: a numerical study using DEM, in AIP Conf Proc (2013), Vol. 1542, pp. 943-946

[3] J. Cordelair, P. Greil, Journal of Materials Science 39, 1017 (2004)

[4] A. Kumar, B. Khusid, Z. Qiu, A. Acrivos, Physical review letters 95, 258301 (2005)

[5] I. Proudman, J. Pearson, Journal of Fluid Mechanics 2, 237 (1957)

[6] E. Guazzelli, J.F. Morris, A physical introduction to suspension dynamics, Vol. 45 (Cambridge University Press, 2011)

[7] B. Metzger, M. Nicolas, E. Guazzelli, Journal of Fluid Mechanics 580, 283 (2007)

[8] F. Pignatel, M. Nicolas, E. Guazzelli, Journal of Fluid Mechanics 671, 34 (2011)

[9] M. Yang, S. Li, J.S. Marshall, Journal of Aerosol Science 90, 154 (2015)

[10] S. Chen, S. Li, W. Liu, H.A. Makse, Soft matter 12, 1836 (2016)

[11] I.M. Jánosi, T. Tél, D.E. Wolf, J.A. Gallas, Physical Review E 56, 2858 (1997) 